\title{
Preface to Special Issue for Panos Macheras
}

\author{
Robert R. Bies ${ }^{1}$
}

Received: 25 March 2019/Accepted: 27 March 2019/Published online: 30 March 2019

(C) Springer Science+Business Media, LLC, part of Springer Nature 2019

I am honored to write this introductory preface to this special tribute issue honoring the work and career of Dr. Panos Macheras of the University of Athens. His achievements and contributions span many areas and are highlighted in the article that follows this preface. This special issue of the Journal of Pharmacokinetics and Pharmacodynamics comprises one tutorial, two reviews and five regular articles. John Ioannidis, of Stanford University, highlights issues of reproducibility in pharmacokinetics from his perspective as author of the PLOS article "Why Most Research Findings are False" [1]. Laszlo Endrenyi consolidates recent discussions regarding bioavailability of highly variable drugs across multiple regulatory agency perspectives and synthesizes the current state of understanding. Athanassios Iliadis highlights matrix approaches to structural identifiability and sensitivity to model parameters through a tutorial. The five original research articles encompass key aspects of pharmaceutical sciences and pharmacometrics that relate to the capture of enzyme correlations when incorporating information into PBPK model frameworks (Nicola Melillo, Amin Rostami), highlights fundamental principles that relate to drug-drug interactions and pharmacogenomic differences (Les Benet), and considers the application Monte Carlo approaches to problems of drug delivery (Kosmidis). The implementation of Bayesian methods are explored using both the STAN and the GNU MCSIM platforms (Sarimveis, Bois et al.) to recapitulate a PBPK model for diazepam. Finally, Pillai and Freedman present an alternative approach to parameter estimation that leverages the invariance principle of ordinary differential equations as applied to nonlinear dynamic systems using chaos synchronization. Altogether, these articles provide a fine tribute to the career and contributions of Panos Macheras.

\section{References}

1. Ioannidis JPA (2005) Why most published research findings Are false. PLoS Med 2(8): e124. https://doi.org/10.1371/journal.pmed. 0020124
Robert R. Bies

robertbi@buffalo.edu

1 University at Buffalo - The State University of New York, Buffalo, NY, USA 